\title{
Response to the comments by Cassel et al.
}

\author{
G. J. H. Dumont • R. J. Verkes • J. K. Buitelaar • \\ J. M. A. van Gerven
}

Received: 25 July 2008 / Accepted: 28 July 2008 /Published online: 2 September 2008

(C) The Author(s) 2008. This article is published with open access at Springerlink.com

In response to Cassel et al., we clearly agree that our findings relate only to our employed blood alcohol concentration (BAC) of $0.56 \mathrm{mg} / \mathrm{ml}$. Different ethanol concentrations may indeed induce different effects, and research in animals such as that reported by Cassel et al. (2005) suggest that higher doses may induce more dramatic interactions. At the same time, it is unfeasible to examine every possible combination of ethanol levels and 3,4methylenedioxymethamphetamine (MDMA) doses. Although our design is inevitably a model, we believe that it is appropriate for the estimation of the effects of recreational drug use by humans for the following reasons.

We employed an ethanol clamp that provides a continuous steady-state BAC of approximately $0.6 \mathrm{mg} / \mathrm{ml}$, which is a radically different approach from the single oral administration of alcohol as employed in the previous studies mentioned by Cassel et al. Although our target BAC reflects the peak achieved after 2-3 alcoholic drinks, due to the continuous infusion of alcohol over $3 \mathrm{~h}$ to maintain this $\mathrm{BAC}$, the total amount of alcohol administered represents a much larger intake of alcoholic drinks corresponding to

Supported by a grant of ZonMW (31000062).

G. J. H. Dumont • R. J. Verkes · J. K. Buitelaar

Unit for Clinical Psychopharmacology and Neuropsychiatry

(UCPN), Department of Psychiatry,

University Medical Centre St Radboud,

Nijmegen, The Netherlands

J. M. A. van Gerven

Centre for Human Drug Research (CHDR),

Leiden, The Netherlands

G. J. H. Dumont $(\bowtie)$

University Medical Centre Nijmegen,

P.O. Box 9101, 6500 Nijmegen, The Netherlands

e-mail: G.J.H.Dumont@psy.umcn.nl almost one bottle of wine, a relevant dose of ethanol which is comparable to the amounts as cited by Cassel et al. in relation to recreational drug use. As recreational alcohol users are expected to spread their alcohol use over the night, our design, thus, represents continuous moderate use of alcohol. A single high dose of oral alcohol (based on total drinks consumed during or around MDMA exposure), on the other hand, will show a rapid increase and a steady decline in BAC, resembling binge drinking. This will induce larger peak effects compared to the effects of our more steady infusion of alcohol. It is unknown whether binge drinking or moderate continuous drinking is more prevalent in MDMA users, and our study represents only the latter pattern of alcohol use. Cassel et al. rightly point to the lack of evidence regarding the real world drinking behavior and achieved BAC by recreational drug users combining MDMA and ethanol, and we welcome further research regarding these uncertainties.

Next, Cassel et al. cites the study by Hernandez Lopez et al. (2002) who achieved a higher BAC (employing a single oral alcohol dose) and reported an increase in MDMA concentration after ethanol coadministration (compared to the MDMA only condition), an effect which we did not find. This report indeed shows a significant increase in MDMA peak levels (Cmax) but not for total MDMA concentrations (AUC0-6 h). This is in line with our expectations and suggestions regarding the difference in ethanol administration route and resulting kinetics. Hernandez-Lopez et al. (2002) also reported a decrease in BAC after ethanol administration, which we did not address in our report. A subsequent evaluation of our data indicated that after MDMA coadministration, significantly more ethanol was needed to maintain the same stable levels of BAC compared to the ethanol only condition (effect of drug condition on infusion rates over time: $F(1,11)=8.537, p=$ 
0.014), in line with the report by Hernandez-Lopez et al. (2002).

Cassel et al. also implies that our results differ from previous human studies by Hernandez-Lopez et al. (2002) and Kuypers et al. (2006), showing a dissociation between subjective and objective measures of sedation and psychomotor function. However, we also report this dissociation. Furthermore, although Hernandez-Lopez et al. (2002) did note a lengthening of euphoria after coadministration of ethanol and MDMA compared to MDMA alone, this effect did not reach the level of statistical significance. Thus, our results do not clearly seem to disagree with those of previous studies, even if BACs were higher than in our study, as in the case of Hernandez-Lopez et al. (2002).

In conclusion, we do not agree that our approach "most probably misses the reality of what's happening." Rather, we are of the opinion that our method represents social drinking behavior that users would expect to be safe and socially acceptable, and interactions with MDMA would, hence, be very relevant, particularly for driving related behavior. No research model can capture all possible realworld behaviors of combined drinking and drug use, and we agree that our model still is a crude estimation of real world drinking behavior, which likely shows a more fluctuating BAC and may affect cognitive function differently (Pohorecky and Brick 1988). However, administration of a single high dose of ethanol may be an even cruder abstraction of real world drinking behavior than relatively constant levels over time. Irrespective of the above, we do agree that higher BACs may yield different outcomes and may provide important data regarding health risks of recreational (binge) drug use. We, thus, welcome future research regarding these issues, although tolerability and ethical issues may hamper the use of much higher doses of ethanol in humans.

Open Access This article is distributed under the terms of the Creative Commons Attribution Noncommercial License which permits any noncommercial use, distribution, and reproduction in any medium, provided the original author(s) and source are credited.

\section{References}

Cassel JC, Riegert C, Rutz S, Koenig J, Rothmaier K, Cosquer B et al (2005) Ethanol, 3,4-methylenedioxymethamphetamine (ecstasy) and their combination: long-term behavioral, neurochemical and neuropharmacological effects in the rat. Neuropsychopharmacology 30:1870-1882

Hernandez-Lopez C, Farre M, Roset PN, Menoyo E, Pizarro N, Ortuno J et al (2002) 3,4-methylenedioxymethamphetamine (ecstasy) and alcohol interactions in humans: Psychomotor performance, subjective effects, and pharmacokinetics. J Pharmacol Exp Ther 300:236-244

Kuypers KP, Samyn N, Ramaekers JG (2006) MDMA and alcohol effects, combined and alone, on objective and subjective measures of actual driving performance and psychomotor function. Psychopharmacology (Berl) 187:467-475

Pohorecky LA, Brick J (1988) Pharmacology of ethanol. Pharmacol Ther 36:335-427 